\title{
Cyclophosphamide Pulse Therapy in Severe Refractory Crohn's Disease: A Retrospective Multicenter Case Series
}

\author{
Florian Bär ${ }^{a}$ Thomas Krause ${ }^{b}$ Andreas Stallmach ${ }^{c}$ Niels Teich ${ }^{d}$ \\ Christian Maaser $^{\mathrm{e}}$ Jochen Maul $^{f}$ Ulf Helwig $^{g}$ Klaus Fellermann $^{a}$ \\ Jürgen Büning ${ }^{a}$ on behalf of the German IBD Study Group
}

${ }^{a}$ Department of Internal Medicine I, University Hospital Schleswig-Holstein, Lübeck, ${ }^{b}$ Gastroenterology Opernstrasse, Kassel, ' Department of Internal Medicine IV (Gastroenterology, Hepatology, Infectious Diseases), University Hospital of Jena, Jena, ${ }^{d}$ Practice for Digestive and Metabolic Diseases, Leipzig, ${ }^{~}$ Department of Internal Medicine, Hospital Lüneburg, Lüneburg, ${ }^{f}$ Charité Medical School, University of Berlin, Berlin, and ${ }^{9}$ Practice of Internal Medicine, Oldenburg, Germany

\section{Keywords}

Cyclophosphamide · Pulse therapy · Refractory Crohn's

disease $\cdot$ Retrospective multicenter case series

\begin{abstract}
Background and Aims: In Crohn's disease (CD) patients still remain refractory to current regimens, including biologicals. Previous data from small single-center studies indicated cyclophosphamide pulse therapy (CPT) to be effective for induction of remission at least in steroid-refractory cases. The aim of the present study was to study the efficacy and safety of CPT in mainly tumor necrosis factor (TNF)-refractory complicated CD patients. Methods: Patients with refractory CD undergoing CPT were identified in 13 centers of the German IBD Study Group and retrospectively registered. In total, 41 patients (12 male, 29 female, median age 36 years, range 18 72 years) were included for analysis. Seventy-eight percent of these had previously been treated with thiopurines and $90 \%$ had previously received anti-TNF antibodies. Former steroid treatment was found throughout the cohort. $\boldsymbol{R e}$ sults: Patients received a median number of $5(1-13)$ pulses
\end{abstract}

() 2017 S. Karger AG, Basel

E-Mail karger@karger.com www.karger.com/iid every 28 (13-54) days in a period of $120(12-411)$ days. A median dose of $766(600-1,200) \mathrm{mg}$ and a median cumulative dose of 4,500 $(750-9,750) \mathrm{mg}$ was given. A clinical response (reduction in the Harvey-Bradshaw Index $[\mathrm{HBI}] \geq 2$ points) was found in $68 \%$ of the patients and clinical remission (HBI $<5$ points) in $32 \%$. Steroids could be reduced from 31 to $12 \mathrm{mg}$ per day over all patients. Side effects were recorded in $71 \%(n=29)$ of the patients. Three patients terminated CPT due to side effects. No patient died. Conclusion: Our data point to CPT as a therapeutic alternative for induction of remission in patients with severe refractory courses of $C D$ including TNF antagonists. CPT might serve as bridging for maintenance treatment.

(c) 2017 S. Karger AG, Basel

\section{Introduction}

Crohn's disease (CD) is characterized by a chronic intestinal inflammation with intermittent flares. More than half of the patients still suffer from disabling disease 5 years after diagnosis [1]. Although the medical armamen- 
tarium continuously expands, some patients remain refractory to current therapeutic strategies such as systemic steroid treatment, classic immunosuppressants, or treatment with antibodies against different components of the inflammatory cascade. The need for surgery in CD patients has decreased in recent decades, but still one-fifth require major surgery within 5 years after diagnosis [2]. However, in some patients surgical options are unsuitable, as in $\mathrm{CD}$ of the upper gastrointestinal tract or long segment involvement of the small bowel.

Systemic steroids remain the mainstay of therapy in severe attacks of $\mathrm{CD}$, but at least $30 \%$ of patients show a refractory disease course [3]. The use of the tumor necrosis factor (TNF) antagonists infliximab and adalimumab substantially improved the outcome in $\mathrm{CD}$, though controlled trials addressing the particular cohort of steroid-refractory $\mathrm{CD}$ patients are lacking. The available data demonstrate a primary nonresponse to TNF antagonists in one-third of CD patients [4-7]. Further therapeutic options were opened by the introduction of vedolizumab and ustekinumab, monoclonal antibodies against $\alpha 4 \beta 7$ integrin and interleukin $12 / 23$ p 40 . With respect to the TNF failure cohort of CD patients, retrospective data of real-world experience showed an initial clinical response to vedolizumab in about $60 \%$ and remission rates at week 54 between 21 and 25\% [8]. A response to ustekinumab was demonstrated in $58 \%$ of patients [9]. The relevant group of nonresponding patients necessitates therapeutic alternatives for induction of remission in severe flares of the disease.

Since the 1960s, the potential of cyclophosphamide as an immunosuppressive agent in the treatment of inflammatory disorders is known. Regarding CD, it has been used as an essential part of autologous hematopoietic stem cell transplantation (HSCT), as recently studied in the ASTIC trial. Despite promising high initial response rates, the final results of this trial showed absence of clinical activity in only $34 \%$ of the severely affected multirefractory CD patients 1 year after the HSCT [10]. However, the high doses of cyclophosphamide used were associated with significant toxicity and even mortality.

By now, cyclophosphamide pulse therapy (CPT) is the therapeutic standard in systemic vasculitis such as systemic lupus erythematosus, vasculitic forms of rheumatoid arthritis, and granulomatosis with polyangiitis [11]. The pulse concept was developed to reduce the cumulative doses of cyclophosphamide and to minimize the corresponding side effects. Several years ago, two small and uncontrolled single-center studies studied the efficacy of CPT at least in steroid-, but not TNF-refractory CD [12, 13]. A clinical response to a median of 4 monthly pulses

Table 1. Demographic characteristics of the patients

\section{Sex \\ L1 \\ L2 \\ L3 \\ L4 \\ B1 \\ B2 \\ B3 \\ B4}

Male

Female

Mean age, years (range)

Montreal classification

Current smoker

Previous surgery

Resections among operated patients

Small bowel resection

Ileocecal resection

Colon resection

Fistulas present

Prior medication

Systemic steroids

Thiopurines

Methotrexate

Tacrolimus

Cyclosporine

Ustekinumab

Tumor necrosis factor antibodies

$>1$ tumor necrosis factor antibody
$12 / 41(29.3 \%)$

$29 / 41(70.7 \%)$

$36(18-72)$

$3 / 41(7.3 \%)$

$8 / 41(19.5 \%)$

$22 / 41(53.7 \%)$

$11 / 41(26.8 \%)$

$13 / 41(31.7 \%)$

$19 / 41(46.3 \%)$

$12 / 41(29.3 \%)$

$10 / 41(24.4 \%)$

$6 / 41(14.6 \%)$

$23 / 41(56.1 \%)$

$14 / 23(61 \%)$

$8 / 23(35 \%)$

$11 / 23(48 \%)$

$3 / 41(7.3 \%)$

$41 / 41(100 \%)$

$32 / 41(78.0 \%)$

$18 / 41(43.9 \%)$

$4 / 41(9.8 \%)$

$3 / 41(7.3 \%)$

$1 / 41(2.4 \%)$

$37 / 41(90.2 \%)$

$27 / 37(73 \%)$

Values are presented as $n(\%)$ unless indicated otherwise.

of cyclophosphamide was observed in $>80 \%$ of the overall 31 patients in both studies.

Obviously the cohorts of $\mathrm{CD}$ patients undergoing HSCT in contrast to CPT are not comparable with respect to the severity of CD or medical history. Nevertheless, $\mathrm{CPT}$ might be a reasonable and rather safe option even for those CD patients with a severe steroid- and TNFrefractory flare not amenable for surgery. The present study used a retrospective multicenter approach in the German IBD Study Group (GISG) to unravel the impact of CPT in multirefractory CD.

\section{Methods}

The study centers for this retrospective study, i.e., medical universities, non-university hospitals, and gastroenterology practices, were recruited by the GISG, which emerged in November 2008 from a joint initiative of the German Working Group on Inflammatory Bowel Diseases and the IBD Competence Network. Pa- 
Table 2. Dose of cyclophosphamide and concomitant medication

\begin{tabular}{lcrr}
\hline & Minimum & Maximum & Median \\
\hline Cumulative dose of cyclophosphamide & & & \\
mg & 750 & 9,750 & 4,500 \\
mg/kg body weight & 12 & 130 & 57 \\
Dose/course & 600 & 1,200 & 766.5 \\
mg & 7.1 & 23.8 & 13.4 \\
mg/kg body weight & 1 & 13 & 5 \\
Cyclophosphamide courses per patient & 13 & 54 & 120 \\
Interval between different courses, days & 12 & 411 & \\
Total duration of cyclophosphamide pulse therapy, days & & & \\
Concomitant medication & $73 \%$ & & \\
$\quad$ Systemic steroids & $10 \%$ & & \\
$\quad$ Azathioprine & $5 \%$ & $2.5 \%$ & \\
$\quad$ Mercaptopurine & & & \\
$\quad$ Methotrexate & & & \\
\hline
\end{tabular}

The characteristics of cyclophosphamide pulse therapy regarding dose, number of courses, interval between cyclophosphamide pulses, and concomitant medication in our study cohort are illustrated.

tients within these centers were identified in case of a CPT due to CD. Charts were retrospectively reviewed, centrally recorded in an anonymous manner by the GISG, and analyzed by the study coordinators. Data were collected from January 2012 to February 2015. The initiation and conception of the CPT was individually determined for each patient by the treating physician without any further study regulations. Thus, all variables of the CPT, e.g., dose, intervals, duration, comedication, and diagnostics, were not influenced by any study requirement.

Thirteen centers contributed to this study. The patients' standard demographic data, baseline characteristics of CD, and medical history were documented (Table 1). The features of the CPT were recorded in detail. The individual doses per infusion ( $\mathrm{mg}$ and $\mathrm{mg} /$ $\mathrm{kg}$ body weight), the total number of infusions, the intervals between the cyclophosphamide pulses (days), the cumulative dose (mg), as well as the treatment period (days) were assessed. Clinical activity of $\mathrm{CD}$ was calculated using the Harvey-Bradshaw Index (HBI). Clinical response was determined as an HBI reduction by $\geq 2$ points and clinical remission as an $\mathrm{HBI}<5$ points. Efficacy was further assessed by the reduction of systemic steroid treatment during the period of CPT. In addition, patients achieving steroid-free remission upon CPT were identified. The reason for termination of CPT was categorized into either "efficacy," "inefficacy," or "side effects." For each patient, the appropriate classification was done by the treating physician. The clinical course was recorded, covering the period of CPT and not more than 3 month thereafter. Surgery related to CD as well as side effects of CD therapy were considered in this period. The results of endoscopic procedures and laboratory tests could not be registered systematically and were consequently not analyzed.

\section{Statistical Analysis}

Results are described as mean, median, and range. Statistical differences were calculated using the Wilcoxon rank test and were considered to be significant at the $p<0.05$ level. Results were calculated using the SPSSWIN program.

Cyclophosphamide in Crohn's Disease

\section{Results}

\section{Patient Characteristics}

The patients' baseline characteristics are shown in Table 1. All patients had been diagnosed according to standard endoscopic, histological, and radiological criteria. Infectious complications such as Clostridium difficile, herpes simplex virus, or cytomegalovirus were ruled out throughout the cohort. Six of the patients (15\%) were current smokers, and 23 patients (56\%) had previously been operated (for type of resection please refer to $\mathrm{Ta}$ ble 1). A fistulizing course of the disease was found in 13 of the patients (32\%), and 3 patients (7\%) suffered from active fistulas at the beginning of the cyclophosphamide treatment. The medical history is depicted in detail in Table 1.

\section{Cyclophosphamide Pulse Therapy}

Patients received a total dose of cyclophosphamide ranging from 750 to $9,750 \mathrm{mg}$ (median 4,500 $\mathrm{mg}$ ). The dose per course ranged from 600 to $1,200 \mathrm{mg}$ (median $767 \mathrm{mg}$, median $13.4 \mathrm{mg} / \mathrm{kg}$, range $7.1-23.8 \mathrm{mg} / \mathrm{kg}) . \mathrm{Pa}-$ tients received a total of $1-13$ courses of CPT (median 5 courses). The interval between courses ranged from 13 to 54 days (median 28 days). The total duration of the cyclophosphamide treatment was a maximum of 411 days (minimum 12 days, median 120 days). As shown in Table 2 , all but one patient got a fluid infusion (full electrolyte solution) in parallel to cyclophosphamide infusion, 


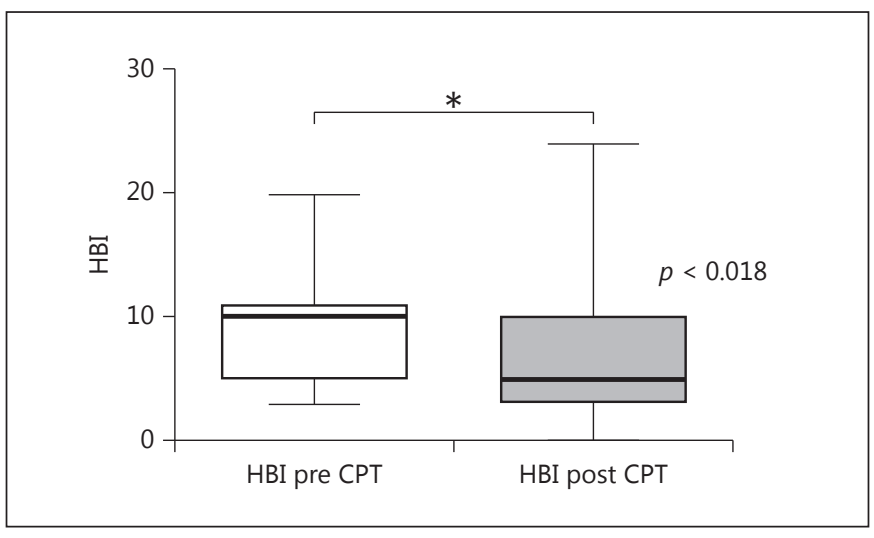

Fig. 1. Clinical activity before and after cyclophosphamide pulse therapy (CPT) expressed by the Harvey-Bradshaw index (HBI). The median HBI at week 0 was 10 points. It dropped to a median of 5 points at the individual end of CPT. This reduction of the median HBI by 5 points was statistically significant $(p<0.018)$ as indicated by the asterisk.

and all patients received sodium-2-mercaptoethanesulfonate (Uromitexan) to prevent hemorrhagic cystitis. Most patients received $1,000 \mathrm{~mL}$ of saline infusion prior and after the administration of cyclophosphamide. Regularly, the dose of mesna was two-thirds the dose of cyclophosphamide. One-third of mesna was given orally to the patient directly with the start of cyclophosphamide infusion as well as 4 and $8 \mathrm{~h}$ thereafter. Antiemetics were given on demand.

Thirty patients $(73 \%)$ received systemic steroids at the start of CPT with a mean dose of $31 \mathrm{mg}$ prednisolone/day (range $0-100 \mathrm{mg} /$ day). With respect to treatment histories, finally all patients were steroid experienced and showed steroid failure. During CPT the steroid dose was adjusted according to the clinical course (see Efficacy of CPT). Four patients $(10 \%)$ were concomitantly treated with azathioprine, with a mean dose of $100 \mathrm{mg}$ of azathioprine/day (range 50-150 mg/day). Mercaptopurine was given in 2 patients (5\%) during the entire course of CPT (37.5 $\mathrm{mg}$ and $100 \mathrm{mg}$ /day). In 1 patient CPT was accompanied by methotrexate $(15 \mathrm{mg} /$ day $)$. No dose adjustment was necessary for either azathioprine, mercaptopurine, or methotrexate. Concomitant treatment with biologicals (TNF antagonists, vedolizumab, ustekinu$\mathrm{mab}$ ) was not found in any of the patients.

\section{Efficacy of CPT}

The HBI was used to calculate the clinical efficacy of the CPT. At the beginning of cyclophosphamide therapy, the median HBI was 10 points (range $1-20$ ). Clinical re-

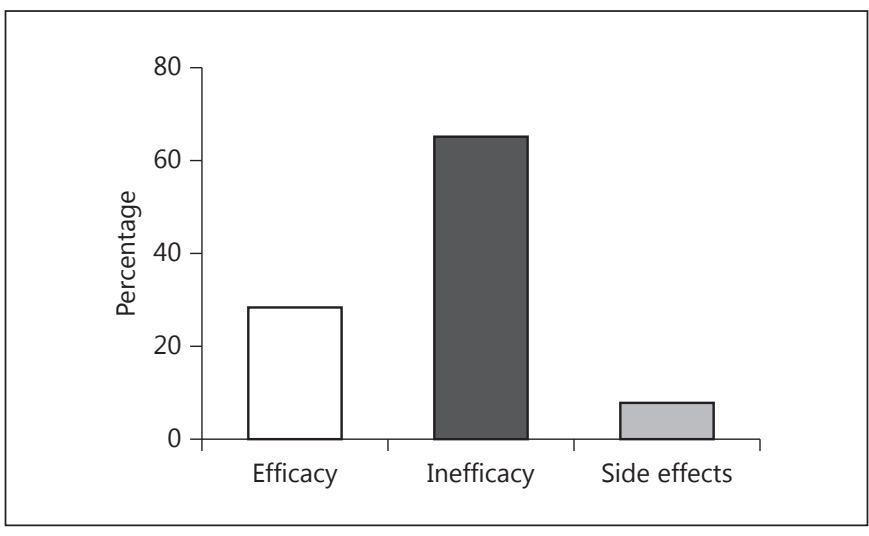

Fig. 2. Assessment of the efficacy of cyclophosphamide pulse therapy (CPT) by the individual treating physician. Clinical efficacy was assessed at the end of the cyclophosphamide period for each patient. The treatment was terminated due to efficacy in $28 \%$ of the patients, while CPT was finally considered ineffective in $65 \%$ of the subjects. In $8 \%$ intolerable side effects were recorded.

sponse to CPT, assumed in case of an HBI reduction of $\geq 2$ points, was detected in $68 \%$ of the patients. One-third (32\%) of the patients went into clinical remission $(\mathrm{HBI}<5$ points) due to CPT. At the individual end of CPT, the median HBI had decreased to 5 points (range 1-24). This reduction in clinical activity according to HBI was significant with a $p$ value $<0.018$ (Fig. 1 ).

Efficacy was additionally assessed by the treating physician at the individual end of the cyclophosphamide period for each patient and categorized in either stop of therapy due to "efficacy," "inefficacy," or "side effects." The treatment was terminated due to efficacy in $28 \%$ of the patients, while CPT was finally considered ineffective in $65 \%$ of the subjects recorded. Intolerable side effects were responsible for the stop of CPT in $8 \%$ of our cohort (Fig. 2).

The median dose of systemic steroids at the beginning of CPT was $31 \mathrm{mg}$ prednisolone/day. During CPT, steroids could be reduced to a median dose of $12 \mathrm{mg}$ prednisolone/day until the individual end of cyclophosphamide (range $0-100 \mathrm{mg}$ ). This reduction was found to be statistically significant with a $p$ value $<0.0005$ (Fig. 3). Four patients who had started with CPT still being on steroids got steroid free during CPT (13\%). Only 2 out of the 11 patients with a clinical response to CPT needed subsequent operation within the follow-up period. In contrast, 15 out of 26 patients without clinical response to CPT underwent surgery in the short term (within a period of 3 months after the last cyclophosphamide course). 


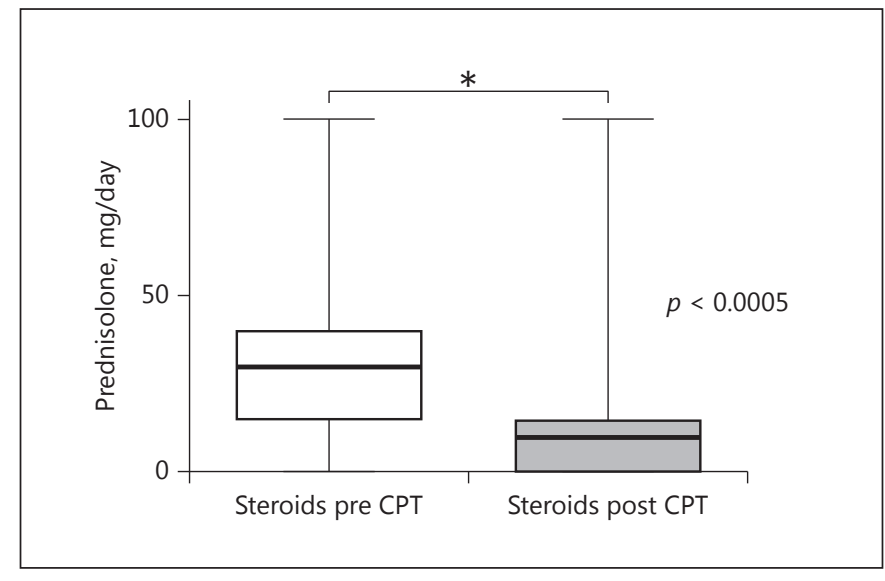

Fig. 3. Reduction of concomitant systemic steroid treatment under cyclophosphamide pulse therapy (CPT). The median steroid dose at the beginning and after $\mathrm{CPT}$ in our cohort is presented. The median steroid dose was $31 \mathrm{mg}$ prednisolone/day at week 0 and dropped to $12 \mathrm{mg}$ prednisolone/day at the individual end of CPT. This reduction by $19 \mathrm{mg}$ prednisolone/day was statistically significant $(p<0.0005)$ as indicated by the asterisk.

\section{Safety Profile of CPT}

In total, 29 of the patients experienced side effects during CPT. Side effects ranged from mild infectious complications (cystitis, esophagitis) to abdominal pain, fever, and sleep disturbances. The most frequent side effects were nausea $(45 \%)$, vomiting (17.5\%), and exhaustion $(12.5 \%)$. Side effects that occurred in up to $5 \%$ of the patients were myalgia, moderate hair loss, headache, dizziness, and restlessness. No serious septic complications were recorded. In 3 patients treatment was terminated due to side effects. None of the patients died. Please refer to Table 3 for further details.

\section{Discussion}

A refractory situation in patients with severe flares of CD still represents a therapeutic challenge, and studies addressing this particular issue are sparse. In our cohort, $90 \%$ of the patients showed TNF failure and all patients were steroid experienced. All patients showed treatment failure to either steroids and immunosuppressants or steroids and biologicals, most of them to all of these substances. More than half of the patients (56\%) underwent surgery in their history, and around one-fourth (24\%) had perianal penetrating disease. In these particularly complex and severe courses of CD, we demonstrated CPT to induce a clinical response in $68 \%$ of the patients. Upon
Table 3. Common side effects under cyclophosphamide pulse therapy

\begin{tabular}{ll}
\hline & Percentage \\
\hline Nausea & 45 \\
Vomiting & 17.5 \\
Exhaustion & 12.5 \\
Fever & 10 \\
Abdominal pain & 10 \\
Sleep disturbance & 7.5 \\
Dizziness & 5 \\
Restlessness & 5 \\
Moderate hair loss & 5 \\
Myalgia & 7.5 \\
Headache & 5 \\
Dysuria, cystitis & 5 \\
Upcoming verruca & 2.5 \\
Tonsillitis & 2.5 \\
Thrombophlebitis & 2.5 \\
Leukopenia & 2.5 \\
Disturbed mobility & 2.5 \\
Disturbed coordination & 2.5 \\
Esophagitis & 2.5 \\
\hline
\end{tabular}

All recorded side effects under cyclophosphamide pulse therapy with a frequency up to $2.5 \%$ are illustrated. It was allowed to record more than one specific side effect for each patient.

CPT, 32\% achieved clinical remission, and therapy was finally determined effective in $28 \%$ of the patients by the treating physician. CPT had to be stopped due to side effects in only $8 \%$, and no patient died under pulsed cyclophosphamide. Here, we provide detailed information on cyclophosphamide given as pulse therapy in a rather large cohort of 41 patients, which might impact therapeutic considerations in critical ill patients with CD.

In active steroid-refractory $\mathrm{CD}$ antibodies against TNF currently represent the treatment of choice. Colombel et al. [14] reported a clinical response to infliximab in $56 \%$ of CD patients, which could be significantly improved to $69 \%$ by adding azathioprine. Under adalimumab, clinical remission at week 26 was achieved in $71.8 \%$ of CD patients, which was not significantly upgraded by combination therapy with azathioprine [15]. As both studies did not explicitly address steroid-refractory cases, the results could not be generally adopted for this particular group of patients. The efficacy of TNF antagonists, either used alone or combined with other immunosuppressants like thiopurines, in severe steroid-refractory courses of $\mathrm{CD}$ will most likely not achieve comparable results, as demonstrated in the SONIC and DIAMOND 
studies. After primary nonresponse to a TNF antagonist, the switch to a second antibody against TNF will be successful in only one-third of patients [16].

A therapeutic alternative in patients who do not respond to TNF antibodies has been opened by the introduction of vedolizumab and ustekinumab. The Gemini III study depicted the efficacy of vedolizumab in CD in a TNF antagonist failure population [17]. Clinical response to vedolizumab at week 10 was observed in $46 \%$ of these patients. In a real-world scenario, vedolizumab was found effective to induce clinical remission at week 54 in $25 \%$ of patients [8]; a further study showed a slightly greater remission rate of $35 \%$ after a median follow-up of 39 weeks [18]. Both cohorts covered $\geq 90 \%$ of patients that were TNF antagonist experienced. Blocking interleukin 12/ 23 p40 by ustekinumab in a TNF antagonist failure population of CD led to a clinical response in $40 \%$ [19]. Without doubt, the introduction of novel biological therapies directed against TNF, $\alpha 4 \beta 7$ integrin, and interleukin 12/ 23 p40 substantially improved the outcomes of CD patients. Nevertheless the demonstrated data clearly point to a relevant population of CD patients resistant to our therapeutic armamentarium, which urges alternatives for induction of remission in severe courses of the disease.

Although the immunological effects might only in part be attributable to cyclophosphamide, high doses of this alkylating substance are the backbone of autologous HSCT. HSCT has been studied as a therapeutic opportunity for multirefractory $\mathrm{CD}$ without reasonable surgical options in two recent trials $[10,20]$. In contrast to 4,500 mg (maximum 9,750 mg) of cyclophosphamide spread over several months in our study, for HSCT around $4 \mathrm{~g}$ of cyclophosphamide were applied on 2 consecutive days during the mobilization phase and another $50 \mathrm{mg} / \mathrm{kg} /$ day for 4 days during the conditioning regimen. In the prospective multicenter trial by Hawkey et al. [10], 23 patients finally underwent HSCT. In the long term (at 1 year), 34\% of the patients were found free of active disease, and only $8 \%$ had reached the primary endpoint of sustained disease remission (a composite primary endpoint of clinical remission, no immunosuppressives/steroids/biologicals, and no evidence of inflammation in endoscopy/radiology). In spite of the rather disappointing efficacy observed by Hawkey et al. [10], the challenging HSCT treatment was accompanied by critical toxicity, and 1 patient even died. The safety of HSCT in CD was directly addressed in the uncontrolled study of Jauregui-Amezaga et al. [20], which enrolled 26 prospectively followed patients. More than $90 \%$ of the patients experienced febrile neutropenia, including two septic shocks. Among noninfectious complications, 57\% developed mucositis and $10 \%$ of the patients had hemorrhagic complications. During the study period of 12 months, various infectious complications were seen, and 1 patient died due to systemic cytomegalovirus infection. The high morbidity and mortality of CD patients related to HSCT in these studies and the apparent need for a subsequent maintenance therapy in most of the patients give rise to some concern and prompt alternative procedures.

The high doses of cyclophosphamide used in HSCT considerably exceed the cumulative doses used in our study as outlined above. In addition, in our study rather small doses of cyclophosphamide were used per pulse (median dose $766 \mathrm{mg}$ ) and were given in intervals of at least 13 days over a period of up to 411 days. Consequently, only minor infectious complications and no septic shocks were reported. None of the 41 patients died under $\mathrm{CPT}$, and only 3 patients had to terminate $\mathrm{CPT}$ due to side effects. This favorable safety profile was analogously observed in the previous studies analyzing CPT in CD $[12,13,21]$. In these studies, the mean cumulative dose of cyclophosphamide was around $3 \mathrm{~g}$ given as monthly pulses of $750 \mathrm{mg}$. Overall, CPT was well tolerated and no lifethreatening side effects, particularly no sepsis with multiorgan failure, occurred. None of the 31 treated patients in these studies died. Ovarian dysfunction and sustained amenorrhea are serious side effects of CPT noticed in HSCT and patients with systemic vasculitis. Ovarian insufficiency is found in 15-27\% of cyclophosphamidetreated vasculitis patients [22]. Persistent ovarian dysfunction would have a dramatic impact on often young women suffering from CD. Fortunately and in accordance with previous $\mathrm{CPT}$ in $\mathrm{CD}$, this side effect was not detected in our patients. As ovarian dysfunction upon cyclophosphamide appears to be dose-related, the avoidance in our study may be attributable to the low cumulative dose, but might also be influenced by the short follow-up of the patients. The same might be true for secondary malignancies such as bladder cancer, leukemia, or lymphoma. With respect to adverse events under cyclophosphamide, the "low-dose" pulse regime, as used in our study, definitely appears favorable compared to HSCT. This might suggest a considerably lower efficacy in the treatment of CD inflammation.

Remarkably, the first studies dealing with CPT in CD showed promising high efficacy rates $[12,13,21]$. Clinical remission upon CPT was reported in $67-81 \%$ of the patients, was rapidly achieved, and lasted for 16-19 months $[12,13]$. In our multicenter study, we were not able to reproduce this excellent efficacy of CPT. Though a clini- 
cal response was found in $68 \%$ of the patients, clinical remission according to an $\mathrm{HBI}<5$ points was achieved in only $32 \%$. We feel confident to have valid results, with around one-third of the patients entering remission upon CPT. The significant steroid-sparing effect under CPT further supports its value and some patients, though a minority, achieved steroid-free remission. Beside the general limitations of a retrospective analysis, we can only speculate upon the cause for the lower efficacy rate of CPT in our study in contrast to the previous ones. The cumulative dose of cyclophosphamide was even higher in the present study (median $4.5 \mathrm{~g}$ vs. $\leq 3 \mathrm{~g}$ ), while the dose per pulse (mean $\approx 750 \mathrm{mg}$ ) and pulse intervals (monthly pulses) were similar. As far as documented, the key phenotypic feature that differs and might have influenced the outcome is a history of TNF antagonist therapy. In the present study, the number of patients with TNF antagonist failure obviously exceeded those of the older studies (90 vs. 19/20\%) [12, 13]. Studies with other CD therapies such as vedolizumab similarly found a worse outcome for the TNF-experienced patients [17], which might indicate a particularly complex CD population.

Major limitations result from the retrospective design of our study. Efficacy data such as clinical response or remission cannot directly be compared to results obtained from prospective controlled trials. Furthermore, the retrospective character prompted shortcomings of outcome variables recorded particularly in the CRP, calprotectin, and endoscopic findings. The lack of these objective parameters might have hampered the interpretation of our results regarding the efficacy of CPT. We had no explicit specification for the indication of CPT and cyclophosphamide as pulse therapy was given in an uncontrolled heterogeneous manner with respect to dosing, intervals, duration, and comedication. Thus, we do not feel confident to give a clear-cut recommendation towards the optimal design of CPT in CD patients. Furthermore, our data did not allow identification of a particular phenotype or subgroup of CD patients that might ideally respond to $\mathrm{CPT}$. We are not able to give valid answers concerning the influence of CPT on specific extraintestinal manifestations or the fistulizing activity of CD. Previous studies, however, speculated on a beneficial effect in patients with extraintestinal manifestations such as pyoderma gangrenosum.

Nevertheless, we herein present the largest cohort of complex refractory $\mathrm{CD}$ patients undergoing $\mathrm{CPT}$. In $\mathrm{CD}$ patients with previous failure of immunosuppressants, steroids, and TNF antagonists not amenable for surgery, CPT clearly demonstrated anti-inflammatory efficacy.
Based on our present data, a successful use of CPT for induction of remission might be expected in about onefourth to one-third of patients with an acceptable risk profile. Keeping in mind the high burden of cyclophosphamide-associated morbidity and mortality in HSCT, $\mathrm{CPT}$ might be a therapeutic alternative to minimize side effects. A multirefractory CD patient without reasonable surgical options and severe inflammatory activity might be a candidate for CPT to rapidly achieve a response. Ideally, once a stable disease situation is entered, the CPT should be succeeded by a less invasive maintenance treatment as far as available. In future projects, the design of $\mathrm{CPT}$ will have to be optimized in terms of dosing, intervals, and duration to improve its efficacy without loss of its favorable risk profile. Furthermore, the identification of patients who might be ideal candidates for CPT (e.g., in contrast to HSCT) will be of pivotal interest.

\section{Statement of Ethics}

The study was conducted according to the Declaration of Helsinki and was approved by the local ethics committee (University of Lübeck, 11-165).

\section{Disclosure Statement}

The authors declare that no financial or other conflict of interest exists in relation to the content of this article.

References

1 Dias CC, Rodrigues PP, da Costa-Pereira A, Magro F: Clinical prognostic factors for disabling Crohn's disease: a systematic review and meta-analysis. World J Gastroenterol 2013;19:3866-3871.

2 Rungoe C, Langholz E, Andersson M, Basit S, Nielsen NM, Wohlfahrt J, Jess T: Changes in medical treatment and surgery rates in inflammatory bowel disease: a nationwide cohort study 1979-2011. Gut 2014;63:16071616.

3 Xu P, He Y, Chen B, Mao R, Tang R, Li M, Chen F, Wu Y, Zeng Z, Chen M: Clinical characteristics and risk factors of refractory Crohn's disease. Zhonghua Yi Xue Za Zhi 2014;94:2982-2987.

4 Hanauer SB: Efficacy and safety of tumor necrosis factor antagonists in Crohn's disease: overview of randomized clinical studies. Rev Gastroenterol Disord 2004;4(suppl 3):S18S24. 
5 Hanauer SB, Sandborn WJ, Rutgeerts P, Fedorak RN, Lukas M, MacIntosh D, Panaccione R, Wolf D, Pollack P: Human anti-tumor necrosis factor monoclonal antibody (adalimumab) in Crohn's disease: the CLASSIC-I trial. Gastroenterology 2006;130:323333.

6 Peyrin-Biroulet L, Deltenre P, de Suray N, Branche J, Sandborn WJ, Colombel JF: Efficacy and safety of tumor necrosis factor antagonists in Crohn's disease: meta-analysis of placebo-controlled trials. Clin Gastroenterol Hepatol 2008;6:644-653.

7 Sandborn WJ, Rutgeerts P, Enns R, Hanauer SB, Colombel JF, Panaccione R, D’Haens G, Li J, Rosenfeld MR, Kent JD, Pollack PF: Adalimumab induction therapy for Crohn disease previously treated with infliximab: a randomized trial. Ann Intern Med 2007;146: 829-838.

8 Stallmach A, Langbein C, Atreya R, Bruns T, Dignass A, Ende K, Hampe J, Hartmann F, Neurath MF, Maul J, Preiss JC, Schmel R, Siegmund B, Schulze H, Teich N, von Arnim U, Baumgart DC, Schmidt C: Vedolizumab provides clinical benefit over 1 year in patients with active inflammatory bowel disease - a prospective multicenter observational study. Aliment Pharmacol Ther 2016;44: 1199-1212.

9 Khorrami S, Ginard D, Marín-Jiménez I, Chaparro M, Sierra M, Aguas M, Sicilia B, García-Sánchez V, Suarez C, Villoria A, Taxonera C, Velasco-Guardado A, MartínezGonzález J, Gisbert JP: Ustekinumab for the treatment of refractory Crohn's disease: the Spanish experience in a large multicentre open-label cohort. Inflamm Bowel Dis 2016; 22:1662-1669.

10 Hawkey CJ, Allez M, Clark MM, Labopin M, Lindsay JO, Ricart E, Rogler G, Rovira M, Satsangi J, Danese S, Russell N, Gribben J, Johnson P, Larghero J, Thieblemont C, Ardizzone S, Dierickx D, Ibatici A, Littlewood T, Onida F, Schanz U, Vermeire S, Colombel JF, Jouet JP, Clark E, Saccardi R, Tyndall A, Travis S, Farge D: Autologous hematopoietic stem cell transplantation for refractory Crohn disease: a randomized clinical trial. JAMA 2015;314: 2524-2534.
11 Hahn BH, McMahon MA, Wilkinson A, Wallace WD, Daikh DI, FitzGerald JD, Karpouzas GA, Merrill JT, Wallace DJ, Yazdany J, Ramsey-Goldman R, Singh K, Khalighi M, Choi SI, Gogia M, Kafaja S, Kamgar M, Lau C, Martin WJ, Parikh S, Peng J, Rastogi A, Chen W, Grossman JM: American College of Rheumatology guidelines for screening, treatment, and management of lupus nephritis. Arthritis Care Res (Hoboken) 2012;64:797-808.

12 Schmidt C, Wittig BM, Moser C, Zeitz M, Stallmach A: Cyclophosphamide pulse therapy followed by azathioprine or methotrexate induces long-term remission in patients with steroid-refractory Crohn's disease. Aliment Pharmacol Ther 2006;24:343-350.

13 Schmidt KJ, Fellermann K, Wellhöner P, Weitz G, Homann N, Herrlinger K, Lehnert H, Ludwig D, Büning J: Clinical trial: cyclophosphamide pulse therapy - a promising therapeutic alternative in refractory Crohn's disease. Aliment Pharmacol Ther 2009;29: 1230-1239.

14 Colombel JF, Sandborn WJ, Reinisch W, Mantzaris GJ, Kornbluth A, Rachmilewitz D, Lichtiger S, D’Haens G, Diamond RH, Broussard DL, Tang KL, van der Woude CJ, Rutgeerts P; SONIC Study Group: Infliximab, azathioprine, or combination therapy for Crohn's disease. N Engl J Med 2010;362: 1383-1395.

15 Matsumoto T, Motoya S, Watanabe K, Hisamatsu T, Nakase H, Yoshimura N, Ishida T, Kato S, Nakagawa T, Esaki M, Nagahori M, Matsui T, Naito Y, Kanai T, Suzuki Y, Nojima M, Watanabe M, Hibi T; DIAMOND study group: Adalimumab monotherapy and a combination with azathioprine for Crohn's disease: a prospective, randomized trial. J Crohns Colitis 2016;10:1259-1266.

16 Gisbert JP, Marín AC, McNicholl AG, Chaparro M: Systematic review with meta-analysis: the efficacy of a second anti-TNF in patients with inflammatory bowel disease whose previous anti-TNF treatment has failed. Aliment Pharmacol Ther 2015;41:613-623.
17 Sands BE, Feagan BG, Rutgeerts P, Colombel JF, Sandborn WJ, Sy R, D’Haens G, Ben-Horin S, Xu J, Rosario M, Fox I, Parikh A, Milch C, Hanauer S: Effects of vedolizumab induction therapy for patients with Crohn's disease in whom tumor necrosis factor antagonist treatment failed. Gastroenterology 2014;147: 618-627.e3.

18 Dulai PS, Singh S, Jiang X, Peerani F, Narula $\mathrm{N}$, Chaudrey K, Whitehead D, Hudesman D, Lukin D, Swaminath A, Shmidt E, Wang S, Boland BS, Chang JT, Kane S, Siegel CA, Loftus EV, Sandborn WJ, Sands BE, Colombel JF: The real-world effectiveness and safety of vedolizumab for moderate-severe Crohn's disease: results from the US VICTORY Consortium. Am J Gastroenterol 2016;111:11471155.

19 Sandborn WJ, Gasink C, Gao LL, Blank MA, Johanns J, Guzzo C, Sands BE, Hanauer SB, Targan S, Rutgeerts P, Ghosh S, de Villiers WJ, Panaccione R, Greenberg G, Schreiber S, Lichtiger S, Feagan BG; CERTIFI Study Group: Ustekinumab induction and maintenance therapy in refractory Crohn's disease. N Engl J Med 2012;367:1519-1528.

20 Jauregui-Amezaga A, Rovira M, Marín P, Salas A, Pinó-Donnay S, Feu F, Elizalde JI, Fernández-Avilés F, Martínez C, Gutiérrez G, Rosiñol L, Carreras E, Urbano A, Lozano M, Cid J, Suárez-Lledó M, Mensa J, Rimola J, Rodríguez S, Masamunt MC, Comas D, Ruíz I, Ramírez-Morros A, Gallego M, Ordás I, Panés J, Ricart E: Improving safety of autologous haematopoietic stem cell transplantation in patients with Crohn's disease. Gut 2016;65: 1456-1462.

21 Stallmach A, Wittig BM, Moser C, Fischinger J, Duchmann R, Zeitz M: Safety and efficacy of intravenous pulse cyclophosphamide in acute steroid refractory inflammatory bowel disease. Gut 2003;52:377-382.

22 Huong DL, Amoura Z, Duhaut P, Sbai A, Costedoat N, Wechsler B, Piette JC: Risk of ovarian failure and fertility after intravenous cyclophosphamide. A study in 84 patients. J Rheumatol 2002;29:2571-2576. 